\title{
Advocating a New Approach to Governing Water, Energy, and Food Security: Testing the Effects of Message Inoculation and Conclusion Explicitness in the Case of the WEF Nexus
}

\author{
Qingjiang Yao (iD, ${ }^{a}$ Praphul Joshi, ${ }^{b}$ Chiung-Fang Chang, ${ }^{c}$ \\ Chelsea McDonalds, ${ }^{d}$ Jason Tran, ${ }^{a}$ Willam Wheeler, ${ }^{c}$ \\ and Shiyue Houe
}

aDepartment of Communication, Lamar University, Beaumont, Texas, USA; ${ }^{b}$ Department of Health and Kinesiology, Lamar University, Beaumont, Texas, USA; 'Department of Sociology, Social Work, and Criminal Justice, Lamar University, Beaumont, Texas, USA; ${ }^{d}$ Baptist Hospital, Beaumont, Texas, USA; eDepartment of Electrical Engineering, Lamar University, Beaumont, Texas, USA

\begin{abstract}
Message sidedness, including its later format inoculation, and conclusion explicitness have been identified by researchers as two prominent message factors that may influence advocating effects. Two-sided messages, which contain both supporting and opposing information about the issue, particularly those containing inoculation components that refute the negative side, are found to be more effective than one-sided messages. Messages with explicit conclusions are also found to be more persuasive than those that let the audience draw the conclusions themselves. This study tested the persuasion effectiveness of message inoculation and conclusion explicitness on a new scientific concept, the water-energy-food (WEF) nexus, of which the public has little knowledge. This study used five randomly assigned groups (total $N=524$ ) and found that messages with explicit conclusions are more persuasive than those with implicit conclusions; however, it found no difference between the effectiveness of one-sided messages and of refutational two-sided messages. The study suggests that a clear conclusion is necessary to communicate the WEF nexus for a better approach to managing the megacrisis of water, energy, and food security.
\end{abstract}

KEYWORDS: Water-energy-food nexus; inoculation theory; conclusion explicitness

The scientific concept of the water-energy-food (WEF) nexus is fairly new. It first emerged in 2011 at the Bonn Nexus Conference, which officially announced water, energy, and food as the three pillars of the nexus (Endo, Tsurita, Burnett, \& Orencio, 2017; Hoff, 2011). Following

CONTACT Qingjiang Yao, PhD • E-mail: qingjiang.yao@lamar.edu • Departmant of Communication, Lamar University, PO Box 10050, Beaumont, TX 77710

() 2018 by Journal of International Crisis and Risk Communication Research. All rights reserved. 
the Bonn Conference, the German federal government launched the website Water, Energy, and Food Security Resource Platform. ${ }^{1}$ The World Economic Forum's (2011) Water Initiative published a book Water Security: The Water-Food-Energy-Climate Nexus. The United Nations and its departments have also adopted the idea quickly. Its Economic and Social Commission for Asia and the Pacific issued the discussion paper "The Status of the Water-Food-Energy Nexus in Asia and the Pacific" (United Nations [UN], 2013b), and its Food and Agriculture Organization published the book An Innovative Accounting Framework for the Food-Energy-Water Nexus: Application for the MuSIASEM Approach to Three Case Studies (UN, 2013a) and the report The Water-Energy-Food Nexus: A New Approach in Support of Food Security and Sustainable Agriculture (UN, 2014). At the same time, the International Institute for Sustainable Development (IISD, 2013) published a report titled The Water-Energy-Food Security Nexus: Towards a Practical Planning and Decision-Support Framework for Landscape Investment and Risk Management. In only a few years, the concept has become central to academic and policy discussions regarding sustainable development and green economy (Biggs et al., 2015).

The idea of the WEF nexus is proposed to solve the conflict between the growth of human population, along with urbanization and globalization, and the constraint of resources. The traditional approach of managing natural resources only focuses on solving crises of a single resource and ignores the possible detrimental effects on other resources. For instance, policy makers favoring the traditional management approach are likely able to foresee that, with the current trend of rapidly increasing demand, global agricultural production will have to increase approximately $70 \%$ by 2050 , and energy production will need to increase approximately $50 \%$ by 2035 . However, they may not foresee the impacts of such increases on water resources, land resources, and climate (Hoff, 2011; UN, 2014). They may also fail to take some critical natural resources into policy consideration. Water, for example, received attention in the green economy discussion only a few years before the nexus concept was launched at the Bonn Conference (Endo et al., 2017; Hoff, 2011). In fact, water, energy, and food are intricately linked. Agriculture uses approximately 70\% of global freshwater, 
food production and transportation account for approximately $30 \%$ of global energy consumption, and energy generation also consumes a large share of water (UN, 2014). The nexus approach, with a systematic view that water, energy, and food security are interdependent and not easily disentangled (IISD, 2013), will improve WEF security and ensure a more consistent and coherent governance by identifying and managing trade-offs and building synergies (UN, 2014).

As significant as it may be, the WEF nexus is still unknown to the public and has no fixed interpretation (Endo et al., 2017). This new scientific concept needs to be promoted to the global public so that key stakeholders can be actively engaged in building a policy framework based on the nexus approach (UN, 2014). This study explores the effectiveness of two traditional but understudied message techniques, inoculation and conclusion explicitness, on audience knowledge, attitude, and behavioral intention toward the WEF nexus. Because the topic is significant but little known to the public, findings of this study can be considered more valid and less influenced by predisposition or other unknown confounding factors.

\section{Literature Review}

Many factors, from the details of message components to the sequence of multiple messages, may have a role in constructing a persuasive message (O'Keefe, 2016). Among these factors, message sidedness, from which the inoculation research was developed, and conclusion explicitness were two that received much attention at the milestone Yale Communication and Attitude Change Program presided by social psychologist Carl Hovland (Hovland, Lumsdaine, \& Sheffield, 1949; Hovland \& Mandell, 1952). They remain intriguing and perplexing aspects of strategic communication research (Belch \& Belch, 2012), in part because research has not reached a consensus how these two message factors impact persuasion effects (O'Keefe, 2016).

\section{Message Sidedness and Inoculation}

Message sidedness addresses whether the persuasive message contains two sides (both positive and negative sides) or just one side (only the 
positive side) of the story. Hovland and colleagues' (1949) pioneer studies in their Yale Project found that two-sided messages were more effective than one-sided messages in changing the attitude of individuals who were initially opposed to the issue. They also found that one-sided messages worked more effectively than two-sided messages with individuals who were already convinced of the position being advocated or with those who were poorly educated.

Many following studies involved two-sided advertising (Crowley \& Hoyer, 1994) and found that two-sided ads tend to be viewed as more credible (Eisend, 2006; O'Keefe, 2016; Pechmann, 1992). Even in celebrity-endorsement ads, two-sided ads are deemed to be significantly more credible and effective than their one-sided counterparts (Kamins, Brand, \& Hoeke, 1989).

However, conclusions on the effectiveness of two-sided ads vary, which is demonstrated in two frequently cited studies. In one study, Pechmann (1992) found that when the opposing point was important (but less important than the promoting point), two-sided ads for a new brand of ice cream led to better brand evaluation, whereas when the opposing point is perceived as trivial, no difference in brand evaluation is caused by one- or two-sidedness. When the opposing point is negatively correlated with the promoting point, the two-sided ads have the greatest effect. Similar studies have even found that two-sided ads' effectiveness increases with greater consumer exposure (Bohner, Einwiller, Erb, \& Siebler, 2003). In another study, however, Crowley and Hoyer (1994) found that for receivers who have a positive preexisting attitude but are aware of the negative information covered in those two-sided ads, the two-sided ads are about as effective as the one-sided ads. If the negative attributes are important to the receivers, the effectiveness of those two-sided ads is lower than it is for their one-sided counterparts.

Inoculation research, first proposed by Hovland's student William McGuire $(1961,1964)$, studies the persuasive effects of a special type of two-sided messages, in which the opposing points are weakened and refuted to resist future exposure to that type of opposing message (Niederdeppe, Gollust, \& Barry, 2014). The persuasion effectiveness of those refutational two-sided messages, according to inoculation researchers (McGuire, 1961; Miller \& Levine, 1996), is much stronger than that of 
their one-sided counterparts. In the inoculation approach, the message first provides some threatening ideas that are strong enough almost to turn the persuadees against the idea. It then equips them with arguments refuting those threatening ideas and defending the original idea (Dillard, 2010). Inoculation researchers have concluded that, in many cases, no matter whether the threatening ideas are refuted, two-sided messages seem to be more persuasive than one-sided messages. However, they also acknowledge that in some studies, one-sided messages appear to be more persuasive (Szabo \& Pfau, 2002).

Several meta-analyses on the effectiveness of one versus two-sided messages have been conducted and have also produced mixed results. Allen (1991) examined 67 experiments and found that when opposing points are raised and refuted (inoculation), two-sided messages are more persuasive than one-sided messages. However, when the opposing points are raised and not refuted, two-sided messages are less persuasive. Eisend (2006) analyzed 34 studies on consumer advertisements from 1960 to 2004 and confirmed that two-sided advertising is generally more effective than one-sided advertising, although the effects are rather small. Such effects of two-sided advertising can be explained by attribution theory, which states that negative information in the messages may cause the audience to perceive the persuaders' motivation to be to tell the truth instead of to make a profit (Burgoon, Pfau, \& Birk, 1995; Eisend, 2006).

O'Keefe (2016) has concluded that no persuasive difference exists between one- and two-sided messages based on his own meta-analyses and synthetic literature review. When refutational (inoculation) and nonrefutational two-sided messages are differentiated, consistent with Allen (1991) but contradictory to Hovland et al. (1949), nonrefutational two-sided messages are less persuasive, whereas refutational two-sided (inoculation) messages can be more persuasive compared with their one-sided counterparts (O'Keefe, 2016). Nonrefutational two-sided advertisements, nevertheless, have no difference compared with their one-sided counterparts. O'Keefe also concluded that none of the possible moderating variables identified in previous studies, such as preexisting attitude, education, or topic involvement, change persuasion effectiveness. 
The WEF nexus approach, which scientists and international organizations view as a better concept with which to manage global water, energy, and food security, is relatively new to the public. Compared with other well-known and rather politicalized scientific topics, such as climate change or stem cell research, the WEF nexus rules out predisposition, a critical confounding factor discussed in the literature. Thus, in this study, we aimed to determine if one-sided and refutational two-sided (inoculation) messages have different effects in promoting the WEF nexus.

\section{Message Conclusion Explicitness}

Conclusion explicitness research investigates whether messages with explicit conclusions (closed ended) and those with implicit conclusions (open ended) have different persuasion effects. Although terminology may vary in this literature (some studies, such as O'Keefe, 1997, used the terms standpoint explicitness, conclusion articulation, and conclusion specificity), closed-ended messages with explicit conclusions contain a direct statement of a conclusion, whereas open-ended messages with implicit conclusions do not (Martin, Lang, \& Wong, 2003). Messages with explicit conclusions generate accurate understanding but may be perceived as threatening or insulting. By contrast, even though messages with implicit conclusions can sometimes generate misunderstanding, they may also be perceived as respectful and inviting and, therefore, as more persuasive (Martin et al., 2003; O’Keefe, 1997).

Hovland also initiated research on conclusion explicitness in his Yale Communication and Attitude Change Program and found that messages with explicit conclusions are more effective in changing audience opinions in the desired direction (Hovland \& Mandell, 1952). Later, some researchers found that such differences between closedended messages with explicit conclusions and open-ended messages with implicit conclusions were often moderated by variables such as education (intelligence), preexisting attitude, audience involvement in the topic, and the complicity of the topic (Armstrong, 2010; Belch \& Belch, 2012). Well-educated or highly ego-involved audiences are more likely to be persuaded by open-ended messages with implicit conclusions, which provide room for them to make up their own minds, 
whereas less educated or ego-involved audiences are more prone to closed-ended messages that provide explicit directions (Kardes, 1988; Sawyer \& Howard, 1991). If the topics of the messages are too complex, however, even well-educated or highly involved receivers may need assistance and be more persuaded by messages with explicit conclusions (Belch \& Belch, 2012). For example, Ahearne, Gruen, and Saxton (2000) found that when the product is complex, such as a CD player, no difference in brand attitude is shown between ads with and without explicit conclusions.

However, again, the findings vary. For instance, Chebat, Charlesbois, and Gelinas-Chebat (2001) found that ads with implicit conclusions generate greater persuasive effects even with low-involvement consumers, as long as those consumers have prior knowledge on the topic. Kardes (1988) found that highly involved consumers exposed to ads with implicit conclusions can generate brand attitude as favorable, as when they are exposed to ads with explicit conclusions, only brand attitudes formed based on ads with implicit conclusions are stronger and more accessible from memory. Other variables, such as the need for cognition and argument quality, have also been identified as moderators of the effectiveness of message conclusion explicitness (Martin et al., 2003).

Even several recent meta-analyses on the role of conclusion explicitness have disagreed with each other. O'Keefe (2016) concluded that little evidence supports the traditionally speculative advantage of messages with implicit conclusions over their counterparts with explicit conclusions. Messages with implicit conclusions are ambiguous and therefore actually encourage receivers to perceive the information as advocating a position either closer (assimilation effect) to or more discrepant (contrast effect) from their position than the messages actually do. In either case, messages with implicit conclusions are less effective than messages with explicit conclusions (O'Keefe, 2016).

Armstrong (2010), nevertheless, believed that O'Keefe's (1997, 2016) conclusion is only valid when no resistance is expected among receivers. When resistance is expected, as in the case of advertising, open-ended messages with implicit conclusions (soft sell) are more advantageous than closed-ended messages with explicit conclusions (hard sell), as traditional advertising wisdom holds. When the audience's freedom 
of drawing conclusions is restricted by ads with explicit conclusions, the audience reasserts its original opinion and rejects persuasion (Armstrong, 2010).

\section{Knowledge, Attitude, and Behavioral Intention}

Research has shown that persuasion effects take place at different levels. Rogers (2003) described five steps of persuasion effects: knowledge, persuasion, decision, implementation, and confirmation. Knowledge means persuadees' awareness and cognitive understanding of the idea; persuasion means the generation of a (favorable or unfavorable) attitude toward it. On the basis of these two steps, decisions will be made to adopt or reject the idea. If the idea is adopted, actions will then be taken (implementation/confirmation). McGuire (1989) maintained that persuasive information needs to climb up 12 levels, from exposure to postbehavioral consolidation, to go through the whole processing procedure in the audience's mind. It loses about half of its audience advancing each level. But in practice, researchers normally use three levels to summarize those cognitive, affective, and conative levels: knowledge, attitude, and behavior (Xu et al., 2010). Most previous studies on persuasion inoculation and conclusion explicitness have focused on one or two levels of effects. This study measures three levels: knowledge, attitude, and behavioral intention toward the WEF nexus. On the basis of the literature reviewed, we plan to test the following hypotheses:

H1: Subjects exposed to a message about the WEF nexus have a higher level of (a) knowledge, (b) supportive attitude, and (c) supportive behavioral intention toward the nexus approach, compared with those who are exposed to no messages on the nexus.

H2: Refutational two-sided message exposure, compared with onesided message exposure, is associated with a higher level of (a) knowledge, (b) supportive attitude, and (c) supportive behavioral intention toward the WEF nexus.

H3: Closed-ended message exposure, compared with open-ended message exposure, is associated with a higher level of (a) knowledge, (b) supportive attitude, and (c) supportive behavioral intention toward the WEF nexus. 


\section{Method}

This study used a $2 \times 2$ factorial design with a control group and was administrated through SurveyMonkey.com. Students from a mid-sized southwestern public university were invited to participate through Web links and e-mails. From December 1 to 24, 2016, 625 responses were collected, of which 524 were used, after incomplete or invalid responses were removed. Among the 524 subjects, ages ranged from 18 to 72 years. Freshmen accounted for $19 \%$, sophomores for $16 \%$, juniors for $20 \%$, seniors for $33 \%$, master's students for $5.4 \%$, doctoral students for $2.7 \%$, and all-but-dissertation students for $2.7 \%$.

\section{Procedure}

Subjects were invited to SurveyMonkey.com to answer a group of questions, which took about 15 minutes to complete. In the middle of the process, a question with five options was embedded. Four options represented four types of message stimuli; one option had no message and served as the control. The five options were designed to be randomly shown, with equal chance, to the subjects.

The four types of messages as stimuli were refutational two-sided open-ended, refutational two-sided closed-ended, one-sided openended, and one-sided closed-ended. All four types were adopted from a short essay on the website of UN Water (n.d.). The essay was about positive evidence supporting the WEF nexus. To produce the two refutational two-sided messages, a paragraph adopted from the UN (2014) was added at the end. The paragraph contained a criticism that the WEF nexus was just "the same old wine in new bottles" (p. 6) and its refutation (inoculation) that the WEF nexus was different from the traditional integrative approaches, which still only explicitly focus on one resource. For the two open-ended messages, the headlines and the ending paragraph both read, "Should we adopt a nexus approach?" For the two closed-ended messages, both the headline and the ending paragraph read, "Let's adopt a nexus approach!"

Subjects could not go back once they passed the page with the stimulus. Among the 524 participants, 111 were exposed to the refutational two-sided open-ended message, 99 to the one-sided open-ended 
message, 99 to the refutational two-sided closed-ended message, 104 to the one-sided closed-ended message, and 91 to no message. Twenty subjects withdrew from participation before being exposed to the stimulus. After receiving the stimulus, four groups of questions measuring subjects' knowledge, supportive attitude, supportive behavioral intention to promote the WEF nexus, and general behavioral intention to reduce WEF consumptions in daily life were administrated.

\section{Dependent Variables}

Knowledge. Five true-false questions based on the messages were used to measure respondents' recall of the information in the message. Answering a question correctly earned 1 point. Thus the index ranged from o to $5(M=1.64, S D=1.44, \alpha=.63)$.

Supportive attitude. Respondents' supportive attitude toward the WEF nexus was measured with an index of six questions, which asked respondents if it was urgent, serious, important, and useful to treat water, energy, and food as a nexus, if the WEF nexus was just another new bottle for old wines in science (reversely coded), and if the respondent was concerned about the WEF nexus. This index ranged from 6 to 30 $(M=20.41, S D=3.97, \alpha=.90)$, with higher values standing for higher levels of attitudinal support for the WEF nexus.

Supportive behavioral intention toward the WEF nexus. Respondents' behavioral intention to promote the approach of the WEF nexus was measured with an index of five items. These items asked respondents to rate the likelihood that they would help promote the WEF nexus approach, solve the problems in the nexus, donate to support the nexus approach, tell people about the nexus approach, or urge people to support the nexus approach. The index ranged from 5 to 25 $(M=14.82, S D=3.52, \alpha=.89)$. Higher values represented a higher level of behavioral intention to promote the WEF nexus.

The indices of the supportive attitude and behavioral intention were adopted and revised from Gastil and Xenos (2010). To better understand persuasion effectiveness at the behavioral level, the following dependent variable was also measured and analyzed.

Supportive behavioral intention in general. Nine items were used to measure how much the respondents would change their life pattern 
to reduce water, energy, and food consumption. The index ranged from 9 to $72(M=39.43, S D=14.27, \alpha=.90)$. Higher values stand for higher levels of behavioral intention to reduce water, energy, and food consumption in daily life.

\section{Manipulation Check}

After being exposed to each type of message or no message, respondents were asked to select if the message (a) provided both positive and negative opinions about the WEF nexus with a clear conclusion, (b) provided just the positive opinions with a clear conclusion, (c) provided both positive and negative opinions with no clear conclusion, (d) provided only positive opinions with no clear conclusion, or (e) was not shown. The actual message types and the subjects' perceptions of the message types were tabulated for a chi-square independent test, $\chi^{2}(16, N=524)=291.0$, $p<$.oo1. The results of the manipulation check are in Table 1.

In each cell of Table 1 , the count and the adjusted standardized residual are reported. Adjusted standardized residuals can be roughly treated as the $z$-values that help tell if, in the cell, the discrepancy between the count and the expected count is statistically significant. Like $z$-values, adjusted standardized residuals with an absolute value of 1.96 or higher refer to statistical significance at the $95 \%$ confidence level, and absolute values of 2.56 or higher stand for statistical significance

TABLE 1 Manipulation Checks on the Message Sidedness and Conclusiveness

\begin{tabular}{lccccc}
\hline $\begin{array}{c}\text { Exposure to/perception } \\
\text { of the message }\end{array}$ & $\begin{array}{c}\text { Closed } \\
\text { ended/two } \\
\text { sided }\end{array}$ & $\begin{array}{c}\text { Closed } \\
\text { ended/one } \\
\text { sided }\end{array}$ & $\begin{array}{c}\text { Open } \\
\text { ended/two } \\
\text { sided }\end{array}$ & $\begin{array}{c}\text { Open } \\
\text { ended/one } \\
\text { sided }\end{array}$ & No message \\
\hline Open ended/two sided & $18,-0.2$ & $15,-1.3$ & $34,3.6$ & $24,1.5$ & $4,-3.9$ \\
Open ended/one sided & $5,-1.2$ & $11,1.1$ & $12,1.3$ & $12,1.7$ & $0,-3.1$ \\
Closed ended/two sided & $49,4.0$ & $49,3.5$ & $30,-1.5$ & $34,0.4$ & $3,-6.6$ \\
Closed ended/one sided & $19,0.8$ & $22,1.4$ & $23,1.4$ & $17,0.2$ & $2,-4.1$ \\
No message & $8,-4.1$ & $7,-14.6$ & $12,-3.7$ & $12,-3.1$ & $82,16.3$ \\
Total & 99 & 104 & 111 & 99 & 91 \\
\hline
\end{tabular}

Note. Entries are the counts and the adjusted standardized residuals from a cross-tabulation analysis. The adjusted standardized residuals can be roughly treated as z-values that indicate the statistical significance of the differences between the counts and the expected counts in the cells (Gerstman, 2015). For the overall tabulation, $X^{2}(16, N=504)=291.0, p<.001$. The two-sided messages contain refutations (inoculations). 
at the $99 \%$ confidence level. As can be seen in Table 1, subjects who were exposed to the open-ended refutational two-sided message were much more likely to report that the message they read provided both positive and negative opinions with no clear conclusion (counts $=34$, adjusted standardized residual $=3.6$ ). Subjects exposed to the closedended two-sided message tended to report the message as providing both positive and negative opinions with a clear conclusion (counts $=49$, adjusted standardized residual $=4.0$ ). Subjects exposed to the onesided messages did not report the distinctive message types correctly. However, as O'Keefe (2003) has argued, manipulation checks on message characteristics do not hurt the validity of the stimulus even if they do not fit the expected pattern. A one-sided message is one sided, no matter how the subjects perceive it.

\section{Results}

Because the hypotheses involve four dependent variables (knowledge, attitude, behavioral intention toward the WEF nexus, and general behavioral intention toward reducing water, energy, and food consumptions), four one-way analyses of variance (ANOVAs) were conducted to see the differences in the four dependent variables across the five groups. Statistically significant differences exist in knowledge, $F(4,499)=14.68, p<.001$; attitude, $F(4,469)=2.48, p=.04$; and general behavioral intention, $F(4,457)=2.41, p=.049$, but not in behavioral intention toward the WEF nexus, across the five groups. Fisher's least significant difference (LSD) post hoc comparisons further revealed the differences between the groups (Table 2).

$\mathrm{H} 1$ hypothesized that reading any version of the message would increase knowledge, supportive attitude, and supportive behavioral intention toward the WEF nexus. The hypothesis was partially supported. As shown in Table 2, the knowledge score of the no-message group was lower than the scores for any of the groups that read a version of the message (Difference no message - open-ended two-sided $=-0.98, p<.001$;

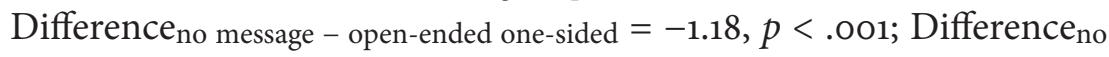
message - closed-ended two-sided $=-1.12, p<.001 ;$ Difference no $_{\text {message }}-$ closedended one-sided $=-1.37, p<.001)$. This significant difference held even 
TABLE 2 Difference in Knowledge, Attitude, Behavioral Intention toward the WEF Nexus, and General Behavioral Intention in Water, Energy, and Food Consumptions Across Five Groups

\begin{tabular}{llcl}
\hline Message version & Knowledge $^{\mathrm{a}}$ & Attitude $^{\mathrm{b}}$ & $\begin{array}{c}\text { General behavioral } \\
\text { intention }\end{array}$ \\
\hline $\begin{array}{c}\text { A (open ended, two } \\
\text { sided) }\end{array}$ & $\mathrm{D}=-0.39^{*}$ & $\mathrm{E}=-4.37^{*}$ \\
$\begin{array}{c}\mathrm{B} \text { (open ended, one } \\
\text { sided) }\end{array}$ & $\mathrm{E}=1.18^{* * *}$ & $\mathrm{D}=-1.71^{* * * *}$ & $\mathrm{D}=-4.39^{*}$ \\
$\begin{array}{c}\text { (closed ended, } \\
\text { two sided) }\end{array}$ & $\mathrm{E}=1.12^{* * *}$ & $\mathrm{E}=-1.31^{*}$ & \\
$\begin{array}{c}\text { (closed ended, } \\
\text { one sided) }\end{array}$ & $\mathrm{A}=0.39^{*}$ & $\mathrm{~B}=4.39^{*}$ \\
$\mathrm{E}$ (no message) & $\mathrm{E}=1.37^{* * *}$ & $\mathrm{~B}=1.71^{* *}$ & \\
& $\mathrm{~A}=-0.98^{* * *}$ & & $\mathrm{~A}=4.37^{*}$ \\
& $\mathrm{~B}=-1.18^{* * *}$ & $\mathrm{~B}=1.31^{*}$ & $\mathrm{~B}=5.13^{*}$ \\
& $\mathrm{C}=-1.12^{* * *}$ & & \\
\hline
\end{tabular}

Note. Entries are mean differences generated from Fisher's LSD post hoc tests of the one-way ANOVAs. With the more conservative Scheffe post hoc tests, only the difference of knowledge scores between the no-message group and the message group still holds. The two-sided messages contain refutations (inoculations).

${ }^{\mathrm{a}} F(4,499)=14.68, \mathrm{p}<.001 .{ }^{\mathrm{b}} F(4,469)=2.48, \mathrm{p}=.04 .{ }^{\mathrm{C}} F(4,457)=2.41, \mathrm{p}=.049$.

${ }^{* * *} p<.001 .{ }^{* *} p<.01 .{ }^{*} p<.05$.

with the more conservative Sheffe's post hoc test, which controls the experiment-wise error rate (Rao, 1998). Meanwhile, the no-message group also had a lower attitude compared with the open-ended one-

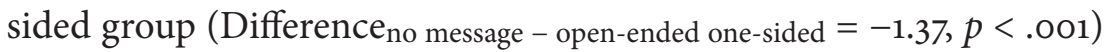
and, surprisingly, a higher general behavioral intention than the two open-ended groups (Difference ${ }_{\text {no }}$ message - open-ended two-sided $=4.37$,

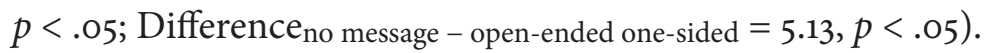

$\mathrm{H} 2$ hypothesized that the refutational two-sided (inoculation) messages, which the audience might perceive as more respectful, were more persuasive than the one-sided messages. That was not supported, as no difference was found between the refutational two-sided groups and the one-sided groups. 
$\mathrm{H} 3$ predicted that closed-ended messages, with explicit conclusions, would be more persuasive than the open-ended messages with implicit conclusions. This hypothesis was partially supported. The open-ended refutational two-sided group gained less knowledge than the closed-end-

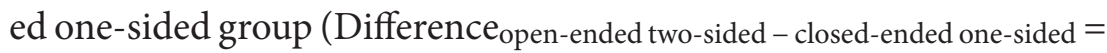
$-0.39, p<.05)$. The open-ended one-sided group, compared with the closed-ended one-sided group, had a lower attitude (Difference open- $_{\text {- }}$ ended one-sided - closed-ended one-sided $=-1.71, p<.01$ ) and general behavioral

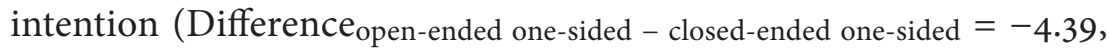
$p<.05)$. Even compared with the no-message group, although the two open-ended groups increased the knowledge scores, they still showed a

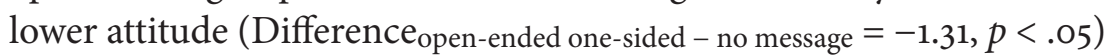
and general behavioral intention toward reducing consumption of water, energy, and food (Difference ${ }_{\text {open-ended two-sided }- \text { no message }}=-4.37, p<.05$; Difference open-ended one-sided - no message $=-5.13, p<.05)$.

\section{Discussion}

Population growth, urbanization, and globalization, among other factors, are creating drastic burdens on indispensable natural resources, particularly water, energy, and agricultural resources. By 2030, it is estimated that demands for water, energy, and food will increase by 40\%, 50\%, and 35\%, respectively (Endo et al., 2017; Hoff, 2011; U.S. National Intelligence Council, 2012). At the same time, water, energy, and food demands are intricately interwoven, and the concept of the WEF nexus is necessary to capture the complex interrelation of these global resources (UN, 2014). The WEF nexus has emerged as a useful concept that takes a central place in the latest discussion of sustainable development and green economy (Biggs et al., 2015).

At the same time, the concept is unfamiliar to the public. On Google Trend, "water-energy-food" is not even a search term, compared with "water security," which generates, on average, two searches a day, or "climate change," which generates, on average, 38 searches a day. In the sample for this study $(N=524)$, on a scale ranging from 1 (I have never heard about the water-energy-food nexus) to 10 (I am very knowledgeable about the water-energy-food nexus), $75.9 \%$ chose 1 and $90.3 \%$ 
chose 4 or lower. The concept of the WEF nexus enables a better understanding and a systematic analysis of the interactions between the natural environment and human activities as well as more coordinated management and use of natural resources (UN, 2014). It is crucial to promote this concept to the public, because public endorsement is key to adopting policy concepts (Endo et al., 2017). The lack of public knowledge regarding the WEF nexus, on the other hand, makes it an ideal topic to test the functions of message inoculation and conclusion explicitness in the effects of strategic promotions, because it rules out the possible moderation of audience predisposition (Armstrong, 2010; O'Keefe, 2016). Unlike heavily politicized scientific topics, such as climate change, the WEF nexus is essentially unknown and clear of political controversy, which eliminates a multitude of possible confounding factors, including audience predisposition.

This study was designed to test if message inoculation (two sided with refutation vs. one sided) and conclusion explicitness (open ended vs. closed ended) make a difference in knowledge, attitude, and behavioral intention toward the WEF nexus as well as general behavioral intention toward improving water, energy, and food security. The study used an experimental design with five randomly assigned groups: one receiving no message and four each receiving an open-ended refutational two-sided, open-ended one-sided, closed-ended refutational twosided, and closed-ended one-sided version of the message, respectively.

As a major point of interest, the data of this study suggest, consistent with O'Keefe (2016) but in contrast to Armstrong (2010) and Belch and Belch (2012), that closed-ended messages with explicit conclusions are more effective in generating desirable results in audience knowledge and attitude toward the WEF nexus as well as the general behavioral intention toward lowering water, energy, and food consumption. Although people tend to think that recognizing an audience's freedom of thinking by providing room for the audience to reach a conclusion themselves may persuade more effectively, it is clear from the evidence generated in this study that messages with an explicit conclusion are more persuasive overall than messages with an implicit conclusion. The evidence was seen in the LSD post hoc tests but not the more conservative Scheffe post hoc tests. However, since the evidence was generated from using 
only one short essay as the stimulus, and the results were consistent with each other, we still deem this finding interesting. Messages with explicit conclusions may appear more confident and therefore heuristically more persuasive. At the same time, conclusion explicitness reduces assimilation effects and/or contrast effects, generating little confusion among the audience (O'Keefe, 2016). By contrast, open-ended messages with implicit conclusions may make both supporters and opponents of the concept of the WEF nexus believe that the message argues for their point of view. Confusion is therefrom produced. The recommendation from that finding is that science communications-at least, communications promoting the WEF nexus-will be better served by including an explicit conclusion.

Message inoculation is not found to make a difference in persuasion effects. Providing criticism of the concept of the WEF nexus and then refuting it does not persuade the audience any more than just arguing for the WEF nexus approach. Researchers have maintained that providing both sides of the story may enhance the credibility of the message in the minds of the audience (Armstrong, 2010; Eisend, 2006) and that refuting a weakened opposing side may enhance the persuasiveness of the supporting side of the message (Dillard, 2010; McGuire, 1964; O'Keefe, 2016; Szabo \& Pfau, 2002). That argument is not supported in this study. The failure to find inoculation effects in this study may be due to the lack of a preexisting favorable attitude toward the WEF nexus, a new topic of megacrisis, or a perception of threat against it among the subjects. Some scholars hold the preexisting favorable attitude or the perception of threats to be the ideal situation for inoculation effects to occur (McGuire, 1964; Niederdeppe et al., 2014). It may also be because the inoculation treatment is not strong enough or because a typical posttest-only method, in lieu of the three-wave method widely used in inoculation studies (first, measuring preexisting attitudes; second, applying inoculation to the experimental group; and third, delivering a subsequent attacking message), was used to measure the inoculation results (Niederdeppe et al., 2014). We are aware, of course, that no difference in communication effects found between the one-sided groups and the refutational two-sided groups in this study means that we fail to support the argument for inoculation messages to be more effective 
than their one-sided counterparts. We cannot, however, simply conclude that no difference exists between inoculation messages and one-sided messages. Further studies on the inoculation effects on new scientific topics need to be accumulated.

It is also worth noting that being exposed to any type of message generates more knowledge on the WEF nexus in the audience's mind. This is the only finding that can be confirmed with the conservative Scheffe post hoc tests. The post hoc analyses also reveal that knowledge is positively correlated with attitude, $r(474)=.22, p<.001$, and general behavioral intention toward reducing water, energy, and food consumption, $r(462)=.14, p<.01$, but not behavioral intention toward promoting WEF nexus. Attitude, however, is substantially positively correlated with both behavioral intention toward the WEF nexus, $r(465)=.66, p<.001$, and general behavioral intention toward reducing water, energy, and food consumption, $r(451)=.47, p<.001$. That provides some evidence for the deficit model, which holds that providing information on scientific topics to the public increases the public's scientific knowledge and therefrom changes its attitude toward science (Sturgis \& Allum, 2004). It is true that groups exposed to a version of the message generate higher levels of supportive attitudes, except the open-ended one-sided group, which generates a lower attitude (Difference ${ }_{\text {open-ended one-sided }- \text { no message }}=-1.31, p<.05$ ) than the nomessage group. But if knowledge on the WEF nexus of all groups exposed to a version of the message increases, knowledge is positively correlated with attitude, and attitude is positively correlated with behavioral intention, it is reasonable to expect that when the informational stimuli turn stronger than just a short essay, public attitude and behavioral intention toward the WEF nexus may increase accordingly.

\section{Conclusion}

In summary, this study confirms that messages with explicit conclusions are more effective in promoting knowledge and attitudes toward the WEF nexus as well as the general behavioral intention toward reducing water, energy, and food consumption, whereas sidedness (inoculation) has no persuasive effects. Providing more information about the WEF 
nexus no doubt increases public knowledge on the nexus approach. When knowledge increases, the public attitude toward the nexus approach may turn more supportive, and the public may have higher levels of intention to do something in support of the nexus approach. The results, although valuable, may be limited in the experimental context with one essay as stimulus. More studies on how message sidedness (inoculation) and conclusion explicitness impact persuasion effects and how persuasion effects travel along the cognitive, affective, and conative levels in the hierarchy of effects should be conducted.

Qingjiang (Q. J.) Yao, $\mathrm{PhD}$, is associate professor at the Department of Communication at Lamar University, Beaumont, Texas. He worked at an educational newspaper in Beijing for six years before pursuing his doctorate in the United States. His research interest currently lies in examining media's persuasive effects in the digital era and particularly on science and health issues.

Praphul Joshi, $\mathrm{PhD}$, is associate professor at the Department of Health and Kinesiology at Lamar University. He is director of the online master's in public health program in the department. He studies stress and health management.

Chiung-Fang Chang, $\mathrm{PhD}$, is associate professor and program director of sociology at Lamar University. She obtained her doctorate in sociology. Her research interests include population studies in China, intermarriage and assimilation among minority populations, racial identity, and fertility behavior of minority women.

Chelsea McDonald graduated from Lamar University with a bachelor of science degree in biology, a bachelor of science degree in psychology, and a minor in chemistry. She is working at Baptist Hospital ER, Beaumont, Texas, and is in the process of applying to medical school.

Jason Tran is pursuing a bachelor's of science in communication with an emphasis on advertising at Lamar University and is anticipating graduation in May 2018. 
William Wheeler is pursuing a bachelor of science degree in sociology at Lamar University.

Shiyue (Jason) Hou is pursuing a master's degree in electrical engineering at Lamar University. He is also an IT support engineer at Lamar University.

\section{ORCID}

Qingjiang (Q, J.) Yao (D) https://orcid.org/oooo-0oo2-0550-4211

\section{Note}

1. http://water-energy-food.org/

\section{References}

Ahearne, M., Gruen, T., \& Saxton, M. K. (2000). When the product is complex, does the advertisement's conclusion matter? Journal of Business Research, 48, 55-62. https://doi.org/10.1016/So148-2963(98)ooo75-7

Allen, M. (1991). Meta-analysis comparing the persuasiveness of one-sided and two-sided messages. Western Journal of Speech Communication, 55, 390-404. https://doi.org/10.1080/10570319109374395

Armstrong, J. S. (2010). Persuasive advertising: Evidence-based principles. New York, NY: Palgrave Macmillan.

Belch, G. E., \& Belch, M. A (2012). Advertising and promotion: An integrated marketing communication perspective (9th ed.). New York, NY: McGrawHill/Irwin.

Biggs, E., Bruce, E., Boruff, B., Duncan, J., Horsley, J., Pauli, N., ... Haworth, B. (2015). Sustainable development and the water-energy-food nexus: A perspective on livelihoods. Environmental Science and Policy, 54, 389-397. https://doi.org/10.1016/j.envsci.2015.08.002

Bohner, G., Einwiller, S., Erb, H.-P., \& Siebler, F. (2003). When small means comfortable: Relations between product attributes in two-sided advertising. Journal of Consumer Psychology, 13, 454-463. https://doi.org/10.1207 /S15327663JCP1304_12 
Burgoon, M., Pfau, M., \& Birk, T. S. (1995). An inoculation theory explanation for the effects of corporate issue/advocacy advertising campaigns. Communication Research, 22, 485-505. https://doi.org/10.1177/0093650950220 04006

Chebat, J.-C., Charlesbois, M., \& Gelinas-Chebat, C. (2001). What makes open vs. closed conclusion advertisements more persuasive? The moderating role of prior knowledge and involvement. Journal of Business Research, 53, 93-102. https://doi.org/10.1016/So148-2963(99)ooo78-8

Crowley, A. E., \& Hoyer, W. D. (1994). An integrative framework for understanding two-sided persuasion. Journal of Consumer Research, 20, 561-574. https://doi.org/10.1086/209370

Dillard, J. P. (2010). Persuasion. In C. R. Berger, M. E. Roloff, \& D. R. Ewoldsen (Eds.), The handbook of communication science (2nd ed., pp. 203-218). Thousand Oaks, CA: Sage.

Eisend, M. (2006). Two-sided advertising: A meta-analysis. International Journal of Research in Marketing, 23, 187-198. https://doi.org/10.1016 /j.ijresmar.2005.11.001

Endo, A., Tsurita, I., Burnett, K., \& Orencio, P. (2017). A review of the current state of research on the water, energy, and food nexus. Journal of Hydrology: Regional Studies, 11, 20-30. https://doi.org/10.1016/j.ejrh.2015.11.010

Gastil, J., \& Xenos, M. (2010). Of attitude and engagement: Clarifying the reciprocal relationship between civic attitudes and political participation. Journal of Communication, 6o, 318-343. https://doi.org/10.1111/j.1460 $-2466.2010 .01484 . x$

Gerstman, B. (2015). Basic biostatistics. Burlington, MA: Jones and Bartlett Learning.

Hoff, J. (2011). Understanding the nexus: Background paper for the Bonn 2011 Nexus Conference. Retrieved from http://wef-conference.gwsp.org/file admin/documents_news/understanding_the_nexus.pdf

Hovland, C. I., Lumsdaine, A. A., \& Sheffield, F. D. (1949). Experiments on mass communication. Princeton, NJ: Princeton University Press.

Hovland, C. I., \& Mandell, W. (1952). An experimental comparison of conclusion drawing by the communicator and by the audience. Journal of Abnormal and Social Psychology, 47, 581-588. https://doi.org/10.1037/hoo 59833

International Institute for Sustainable Development. (2013). The water- 
energy-food security nexus: Towards a practical planning and decisionsupport framework for landscape investment and risk management. Retrieved from http://www.iisd.org/pdf/2013/wef_nexus_2013.pdf

Kamins, M. A., Brand, M. J., \& Hoeke, S. A. (1989). Two-sided versus one-sided celebrity endorsement: The impact on advertising effectiveness and credibility. Journal of Advertising, 18(2), 4-10. https://doi.org/10.1080/00913367 .1989 .10673146

Kardes, F. R. (1988). Spontaneous inference process in advertising: The effects of conclusions omission and involvement on persuasion. Journal of Consumer Research, 15, 225-233. https://doi.org/10.1016/S1057-7408(o8)80 053-8.

Martin, B. A., Lang, B., \& Wong, S. (2003). Conclusion explicitness in advertising: The moderating role of need for cognition (NFC) and argument quality (AQ) on persuasion. Journal of Advertising, 32(4), 57-65. https:// doi.org/10.1080/o0913367.2003.10639148

McGuire, W. (1961). The effectiveness of supportive and refutational defenses in immunizing and restoring beliefs against persuasion. Sociometry, 24, 184-197. https://doi.org/10.2307/2786067

McGuire, W. (1964). Inducing resistance to persuasion: Some contemporary approaches. In L. Berkowitz (Ed.), Advances in experimental social psychology (Vol. 1, pp. 191-229). New York, NY: Academic Press.

McGuire, W. (1989). Theoretical foundations of campaigns. In R. Rice \& C. Atkin (Eds.), Public communication campaigns (2nd ed., pp. 43-65). Newbury Park, CA: Sage.

Miller, M. D., \& Levine, T. R. (1996). Persuasion. In M. Salwen \& D. Stacks (Eds.), An integrated approach to communication theory and research (pp. 261-276). Mahwah, NJ: Lawrence Erlbaum.

Niederdeppe, J., Gollust, S., \& Barry, C. (2014). Inoculation in competitive framing: Examining message effects on policy preferences. Public Opinion Quarterly, 78, 634-655. https://doi.org/10.1093/poq/nfuo26

O'Keefe, D. (1997). Standpoint explicitness and persuasive effect: A metaanalytic review of the effects of varying conclusion articulation in persuasive messages. Argumentation and Advocacy, 34(1), 1-12. https://doi.org/10 $.1080 / 00028533.1998 .11951621$

O'Keefe, D. (2003). Message properties, mediating states, and manipulation checks: Claims, evidence, and data analysis in experimental persuasive 
message effects research. Communication Theory, 13, 251-274. https://doi .org/10.1111/j.1468-2885.2003.tboo292.x

O'Keefe, D. (2016). Persuasion: Theory and research (3rd ed.). Thousand Oaks, CA: Sage.

Pechmann, C. (1992). Predicting when two-sided ads will be more effective than one-sided ads: The role of correlation and correspondent inferences. Journal of Marketing Research, 29, 441-453. https://doi.org/10.2307/3172710 Rao, P. (1998). Statistical research methods in the life sciences. Pacific Grove, CA: Duxbury Press.

Rogers, E. (2003). Diffusion of innovation (5th ed.). New York, NY: Free Press. Sawyer, A., \& Howard, D. (1991). Effects of omitting conclusions in advertisement to involved and uninvolved audiences. Journal of Marketing Research, 28, 467-474. https://doi.org/10.2307/3172786

Sturgis, P., \& Allum, N. (2004). Science in society: Re-evaluating the deficit model of public attitude. Public Understanding of Science, 13(1), 55-74. https://doi.org/10.1177/096366250404269o

Szabo, E. A., \& Pfau, M. (2002). Nuance in inoculation: Theory and applications. In J. Dillard \& M. Pfau (Eds.), The persuasion handbook: Development in theory and practice (pp. 233-258). Thousand Oaks, CA: Sage.

United Nations. (2013a). An innovative accounting framework for the foodenergy-water nexus: Application for the MUSIASEM approach to three case studies. Retrieved from http://www.fao.org/docrep/o19/i3468e/i3468e.pdf

United Nations. (2013b). The status of the water-food-energy nexus in Asia and the Pacific. Retrieved from http://www.unescap.org/sites/default/files /Water-Food-Nexus\%2oReport.pdf

United Nations. (2014). The water-energy-food nexus: A new approach in support of food security and sustainable agriculture. Retrieved from http:// www.fao.org/3/a-bl496e.pdf

UN Water. (n.d.). Water, food and energy. Retrieved from http://www.unwater .org/water-facts/water-food-and-energy/

U.S. National Intelligence Council. (2012). Global trends 2030: Alternative worlds. Washington, DC: Author.

World Economy Forum. (2011). Water security: The water-energy-foodclimate nexus. Washington, DC: Island Press. Retrieved from http://www3 .weforum.org/docs/WEF_WI_WaterSecurity_WaterFoodEnergyClimate Nexus_2011.pdf 
Xu, W., Sun, G., Lin, Z., Chen, M., Yang, B., Chen, H., \& Cao, K. (2010). Knowledge, attitude, and behavior in patients with atrial fibrillation undergoing radiofrequency catheter ablation. Journal of Interventional Cardiac Electrophysiology, 28, 199-207. https://doi.org/10.1007/s10840-010 -9496-2 